\begin{tabular}{ccc}
\hline RECORDS OF PHARMACEUTICAL \\
AND BIOMEDICAL SCIENCES
\end{tabular}

\title{
Recent advances in ambient electrospray ionization technologies
}

\author{
Sara Amer ${ }^{a, b^{*}}$, Walaa Zarad ${ }^{a}$, Heba El-Gendy ${ }^{a}$, Randa Abdel-Salam ${ }^{c}$, Ghada Hadad $^{c}$, Tsutomu \\ Masujima $^{b, \hat{r}}$, and Samy Emara ${ }^{a}$ \\ ${ }^{a}$ Faculty of Pharmacy, Misr International University, Km 28 Ismailia Road, Cairo 11865, Egypt \\ ${ }^{b}$ Quantitative Biology Center (QBiC), RIKEN, 6-2-3 Furuedai, Suita, Osaka 565-0874, Japan \\ ${ }^{c}$ Pharmaceutical Analytical Chemistry Department, Faculty of Pharmacy, University of Suez Canal, Ismailia \\ 41522, Egypt
}

Received on: 11. 03. 2020

Revised on: 15. 04. 2020

Accepted on: 18. 04. 2020

Correspondence Author:

Tel: 01062737274

E-mail address:

sara.m.amer@hotmail.com

\begin{abstract}
Mass spectrometry is one of the most powerful techniques for detection and identification. Due to its sensitivity and selectivity, exact mass of analytes, and in effect, their relative fragments, allow for total structure information. As so, mass spectrometry has now played a major role in branches such as forensics, academic research, environmental studies, and manufacturing quality control. The most recent technological advances have not been to the body of the mass spectrometer, but rather to the first step only, the ion source. The ionization mechanism has been found to impact a plethora analytical results including, but not limited to, the target analytes to be ionized, the relation between sensitivity and ionization efficiency, and the sample inlet flux in relation to analysis time. This mini review focuses on the latest technologies in ambient ionization of mass spectrometry including desorption electrospray ionization, paper spray ionization, laser ablation electrospray ionization, and direct nano-electrospray ionization.
\end{abstract}

Keywords: ambient electrospray ionization; desorption electrospray ionization; paper spray ionization; laser ablation electrospray ionization; direct nanoelectrospray ionization

\section{Introduction}

A standard electrospray ionization (ESI) device is described as having an analyte solution pumped at a low flow rate ( $\mu \mathrm{L}$ scale) through a capillary needle connected to a high potential electric source, with a counter electrode at the orifice of the mass spectrometer (MS) [Ho et al, 2003]. Upon electrical output, a strong electric field is formed and causes. the release of the analyte solution in the form of an aerosol. These droplets formed move through the atmosphere, shrinking in size until free ions, in the form of protonated $(\mathrm{M}+\mathrm{H})+$, deprotonated $(\mathrm{M}-\mathrm{H})-$, or adduct molecular ions, are released and enter the MS orifice under vacuum to be analyzed The 
response of ESI ionization is mainly affected by the mobile phase composition and sample constituents and any co-eluting compounds, thereby causing a matrix effect [King et al, 2000]. This may cause either ion suppression or enhancement, thereby being one of the main drawbacks of ESI. The combination of MS with liquid chromatography (LC) [Chambers et al, 2007, Lee and Kerns, 1999, Van Eeckhaut et al, 2009, Xu et al, 2007] and capillary electrophoresis (CE) [Ramautar et al, 2009, Ramautar et al, 2019, Schappler et al, 2016, Týčová et al, 2017] have greatly reduced co-elution related problems and in effect minimized their effect. And while high resolution MS instruments paired with accurate fragmentation strategies have advanced many fields, such as metabolomics and proteomics, the need for accurate and reliable electronic libraries is still a limiting factor in matching structural information.

The newest branch of ESI technologies, named ambient ionization methods, have overcome the downsides of ESI and allowed for direct analysis of intact samples. These techniques have allowed for analysis methods independent of sample preparation or separation systems, all at ambient pressure and temperature. This mini-review will cover emerging ambient MS methodology and studies, with a focus on desorption electrospray ionization (DESI), paper spray ionization (PSI), laser ablation electrospray ionization (LAESI), and direct nano-electrospray ionization (nESI).

\section{Desorption electrospray ionization}

DESI involves spraying a charged solvent onto a sample deposited on a probe material, such as polytetrafluoroethylene, polymethyl methacrylate, or glass, or is applied directly on the sample surface. The solvent forms a thin layer of liquid on the sample surface, dissolving soluble compounds. Additional solvent then hits the liquid surface and displaces smaller secondary droplets containing the soluble analytes, which follow desolvation and ionization steps into the gas phase, and then enter the mass spectrometer. This method of ionization has been used successfully in many different fields, including clinical applications, food quality control, and forensic science.

Pirro et al. recently described a successful DESIMS method for the analysis of fresh brain tissue smears for lipidomic analysis [Pirro et al, 2017]. They successfully applied their protocol to both human and mouse brain tissues, and produced results containing different lipid classes unique to each sample type, and confirmed compound lipid structures using low energy collision induced dissociation.

Ferreira et al. described a detailed approach for the analysis of individual oocytes and preimplantation embryos with the goal of in situ lipidomic analysis [Ferreira et al, 2020] . The method was successfully applied on cells recovered from mouse, bovine, and swine.

Banerjee et al. proved the usefulness of in cancer research [Banerjee et al, 2017]. Their approach allowed for them to differenciate between benign and cancerous cells of the prostate. Analysis was performed within minutes and identified Kreb's cycle intermediates as distinguishing markers. Due to the high glucose utilization of cancer cells, a glucose to citrate ratio was used to accurately differentiate the cells in $90 \%$ of the specimens. 
As a sub-branch, nano-DESI has proven to be a successful technique for surface sampling. Contrary to its name, the instrumental setup is quite different from that of tradition DESI. Nano-DESI uses two silica caplillaries- the first delivers the solvent to the sample surface, while the second collects and forms a charged, more efficient spray to be fed into the mass spectrometer. The second capillary's spray is independent of the first capillary, and the liquid moves only due to the high voltage between the first capillary and the MS inlet, which cause a solvent bridge between the two capillaries. CardosoPalacios and Lanekoff proved this method useful in the analysis of counterfeit drugs, successfully analyzing fourteen different brands of tablets containing for different active pharmaceutical ingredients and grouping them using principal component analysis [Cardoso-Palacios and Lanekoff, 2016].

\section{Paper spray ionization}

Unlike DESI, PSI utilizes a triangular piece of paper as the sample support. The sample is spotted on the farther corner, then the rear is sprayed with the suitable spray solvent for extraction. The paper is then attached to a clip connected to a high voltage power source; once said high voltage is applied, the paper produces an electrospray-like ionization stream [McBride et al, 2019]. Signal stability depends on the solvent composition, distance of the paper tip from the MS inlet, and paper angle. These parameters, along with other physical properties of the paper used were explored by Bills et al. in the analysis of fentanyl and synthetic cannabinoids [Bills et al, 2018]. PSI has become an attractive analytical technique in the clinical, forensic, and environmental fields.

Jett et al. used the high sensitivity and rapid analysis times of PSI to successfully analyze 130 target compounds at average toxicological cut-off concentrations in selective reaction monitoring mode, all in less than two minutes, from dried blood spots [Jett et al, 2017]. The solvent used was 95:5:00.1 methanol:water:acetic acid and analysis was done on a TSQ Vantage triple quadrupole mass spectrometer.

A comprehensive PSI based analysis of urine was done by Michely et al. to compare the results of this direct ambient ionization method to classical LC-MS methods after sample extraction, precipitation, and conjugation steps [Michely et al, 2017]. After the analysis of 103 intact human samples, their results showed that $73 \%$ of all drugs tested for (777 drugs) were detected using PSI, compared to a maximum identification rate of $88 \%$ from more traditional methods. This was mainly due to either matrix effects or low analyte concentrations. They believe that their results are quite promising due to the rapid analysis, cost effectiveness, and simplicity of their proposed method.

\section{Laser ablation electrospray ionization}

Through modification of DESI, LAESI was developed by separation the desorption and ionization processes at atmospheric conditions [Bartels and Svatoš, 2015]. Desorption is done by a pulsed laser passing across the surface of the sample. This new technology allows for the visualization of solid samples in 2D and 3D. 
The team of van Geenen applied LAESI to characterize synthetic fibers and polymers, a valuable application in the manufacturing industry to determine the value of end product storage and packaging containers [van Geenen et al, 2017]. They successfully analyzed, differentiated, and depicted different forms of polyamide.

A protocol of plant metabolomics was developed through the use of were spatial resolution using LAESI by Etalo et al [Etalo et al, 2018]. Laser was pulsed directly on living plant materials, specifically, petals of Phalaenopsis orchid petals. Ion formation depended on the laser forming small water bubbles on the petal surface upon ablation. Once these bubbles popped, vaporized material is released above the sample surface. An electrospray source sprays the ionization solvent above the petal, which intersects the petal material, ionizes the sample molecules, and then carries it in the MS for analysis.

LAESI was used for confident identification of biomolecules directly from intact biological samples such as soybean seeds, mouse kidneys, and common coleus leaves [Stopka et al, 2019]. The ionization method was coupled to a 21 tesla Fourier transform ion cyclotron resonance mass spectrometer for increased mass resolution a decreased matrix interferences.

\section{Direct nano-electrospray ionization}

While many argue that the mechanism of nESI is similar to that of traditional ESI in regards to spray development, this is not the case [Juraschek et al, 1999]. Briefly, the sample liquid is loaded into a conductant-covered glass capillary and placed in from of the MS orifice. At voltage application, charged compounds move and collect at the capillary tip, forming a "Taylor cone", which then disrupts into a fine jet of charged droplets. These droplets begin quite small in size when compared to traditional ESI primary droplets, and undergo solvent evaporation until their respective Rayleigh limit is achieved, at which droplet fission occurs. This process is repeated until singularly charged analyte molecules are formed, which enter the MS [Amer et al, 2019]. This process is depicted in Figure 1. Advantages of nESI over conventional ESI include minute sample consumption, minimized flow rate and more natural and steady cone stream, a higher tolerance of salt and buffer concentrations, and a more even distribution of charge over all analytes, including non-surface active compounds.

A chip-based nESI sample delivery system was developed by Southam et al. for metabolomic and lipidomic analysis in order to identify metabolic and lipid profiles in biological samples, and in effect, explore biological phenotypes [Southam et al, 2017].

Tsukuia et al. proposed a chip based direct-infusion nESI-MS for the discovery of atractyligenin derivatives as potential markers for green coffee postharvest discrimination [Tsukui et al, 2019]. They successfully discriminated between two different crops of green coffee processed by two different treatments through their chemical profiles. A dual non-contact nESI/nAPCI (nanoatmospheric pressure chemical ionization) source was successfully constructed by Kulyk et al. that simultaneously analyzed polar and non-polar 




Figure 1: Depiction of ionization by nESI. Reprinted with permission from [Amer et al., 2019].

compounds without any sample pretreatment [Kulyk et al, 2019]. The method was successfully applied on bio-fluids and proteins in high buffer concentrations, proving the possible applications in clinical and forensic settings. Volatile organic compounds were identified in human breath in real time by Li et al. through the use of secondary nESI [Li et al, 2017]. In this case, the sample is a direct human breath exhaled into a stanless steel ionization chamber. The ionization solvent was introduced perpendicular to the breath by nESI, and subsequently, carried into the MS. They were able to detect and confirm non-volatile compounds such as sulfate/sulfuric acid and silicate/silicic acid, along with indoor air pollutants as well as ammonia-containg compounds derived from the oral cavity and gastrointestinal bacterial fermentation. The study compared results at different resolving powers, and concluded that only at very high resolving powers $(\geq 60,000)$ could it be possible to achieve accurate non-target analysis.

\section{Conclusion}

Ambient ionization techniques have made great leaps in the sciences of mass spectrometry over the last decade. The ability for analysis without sample preprocessing, chemical microscopy, and lower analysis times are advantages over traditional ionization techniques. This has allowed them to be applied in bioanalytical, environmental, clinical, forensics, and quality control fields. Advances in this field have included sample automation, simultaneous analysis of non-polar molecules, and complex samples. And although matrix effects were of a main concern, advances in the minimization of its effects has led to more sensitive and reliable methodologies, achieving accurate quantitation results. Ambient ionization has become an essential technique in modern MS, and looks to be a persistent technique for future researches. 


\section{References}

Amer S., Zarad, W., El-Gendy, H., Abdel-Salam, R., Hadad, G., Masujima, T., et al. 2019. Direct nano-electrospray ionization tandem mass spectrometry for the quantification and identification of metronidazole in its dosage form and human urine. Royal Society Open Science. 6(11), 191336.

Banerjee S., Zare, R.N., Tibshirani, R.J., Kunder, C.A., Nolley, R., Fan, R., et al. 2017. Diagnosis of prostate cancer by desorption electrospray ionization mass spectrometric imaging of small metabolites and lipids. Proceedings of the National Academy of Sciences of the United States of America. 114(13),3334-9.

Bartels B., Svatoš, A. 2015. Spatially resolved in vivo plant metabolomics by laser ablation-based mass spectrometry imaging (MSI) techniques: LDIMSI and LAESI. Frontiers in plant science. 6,471.

Bills B.J., Kinkade, J., Ren, G., Manicke, N.E. 2018. The impacts of paper properties on matrix effects during paper spray mass spectrometry analysis of prescription drugs, fentanyl and synthetic cannabinoids. Forensic Chemistry. 11,1522.

Cardoso-Palacios C., Lanekoff, I. 2016. Direct analysis of pharmaceutical drugs using nano-DESI MS. Journal of analytical methods in chemistry. 2016.

Chambers E., Wagrowski-Diehl, D.M., Lu, Z., Mazzeo, J.R. 2007. Systematic and comprehensive strategy for reducing matrix effects in LC/MS/MS analyses. Journal of Chromatography B. 852(12),22-34.

Etalo D.W., Díez-Simón, C., de Vos, R.C.H., Hall,
R.D. Laser Ablation Electrospray Ionization-Mass Spectrometry Imaging (LAESI-MS) for Spatially Resolved Plant Metabolomics. In: António C, editor. Plant Metabolomics: Methods and Protocols. New York, NY: Springer New York; 2018. p. 253-67.

Ferreira C.R., Pirro, V., Jarmusch, A.K., Alfaro, C.M., Cooks, R.G. Ambient Lipidomic Analysis of Single Mammalian Oocytes and Preimplantation Embryos Using Desorption Electrospray Ionization (DESI) Mass Spectrometry. In: Shrestha B, editor. Single Cell Metabolism: Methods and Protocols. New York, NY: Springer New York; 2020. p. 15979.

Ho C.S., Lam, C.W.K., Chan, M.H.M., Cheung, R.C.K., Law, L.K., Lit, L.C.W., et al. 2003. Electrospray ionisation mass spectrometry: principles and clinical applications. Clin Biochem Rev. 24(1),3-12.

Jett R., Skaggs, C., Manicke, N.E. 2017. Drug screening method development for paper spray coupled to a triple quadrupole mass spectrometer. Analytical Methods. 9(34),5037-43.

Juraschek R., Dülcks, T., Karas, M. 1999. Nanoelectrospray-More than just a minimizedflow electrospray ionization source. Journal of the American Society for Mass Spectrometry. 10(4),300-8.

King R., Bonfiglio, R., Fernandez-Metzler, C., Miller-Stein, C., Olah, T. 2000. Mechanistic investigation of ionization suppression in electrospray ionization. Journal of the American Society for Mass Spectrometry. 11(11),942-50. Kulyk D.S., Swiner, D.J., Sahraeian, T., BaduTawiah, A.K. 2019. Direct Mass Spectrometry 
Analysis of Complex Mixtures by Nanoelectrospray with Simultaneous Atmospheric Pressure Chemical Ionization and Electrophoretic Separation Capabilities. Analytical Chemistry. 91(18),11562-8. Lee M.S., Kerns, E.H. 1999. LC/MS applications in drug development. Mass spectrometry reviews. 18(3-4),187-279.

Li X., Huang, L., Zhu, H., Zhou, Z. 2017. Direct human breath analysis by secondary nanoelectrospray ionization ultrahigh-resolution mass spectrometry: Importance of high mass resolution and mass accuracy. Rapid Communications in Mass Spectrometry. 31(3),301-8.

McBride E.M., Mach, P.M., Dhummakupt, E.S., Dowling, S., Carmany, D.O., Demond, P.S., et al. 2019. Paper spray ionization: Applications and perspectives. TrAC Trends in Analytical Chemistry. 118,722-30.

Michely J.A., Meyer, M.R., Maurer, H.H. 2017. Paper Spray Ionization Coupled to High Resolution Tandem Mass Spectrometry for Comprehensive Urine Drug Testing in Comparison to Liquid Chromatography-Coupled Techniques after Urine Precipitation or Dried Urine Spot Workup. Analytical Chemistry. 89(21),11779-86.

Pirro V., Jarmusch, A.K., Ferreira, C.R., Cooks, R.G. Ambient Lipidomic Analysis of Brain Tissue Using Desorption Electrospray Ionization (DESI) Mass Spectrometry. In: Wood P, editor. Lipidomics. New York, NY: Springer New York; 2017. p. 187210.

Ramautar R., Somsen, G.W., de Jong, G.J. 2009. CE-MS in metabolomics. Electrophoresis. 30(1),276-91.

Ramautar R., Somsen, G.W., de Jong, G.J. 2019.
CE-MS for metabolomics: Developments and applications in the period 2016-2018. Electrophoresis. 40(1),165-79.

Schappler J., González-Ruiz, V., Rudaz, S. 2016. CE-MS in drug analysis and bioanalysis. Capillary Electrophoresis-Mass Spectrometry (CE-MS).12957.

Southam A.D., Weber, R.J.M., Engel, J., Jones, M.R., Viant, M.R. 2017. A complete workflow for high-resolution spectral-stitching nanoelectrospray direct-infusion mass-spectrometry-based metabolomics and lipidomics. Nature protocols. 12(2),310-28.

Stopka S.A., Samarah, L.Z., Shaw, J.B., Liyu, A.V., Veličković, D., Agtuca, B.J., et al. 2019. Ambient Metabolic Profiling and Imaging of Biological Samples with Ultrahigh Molecular Resolution Using Laser Ablation Electrospray Ionization 21 Tesla FTICR Mass Spectrometry. Analytical Chemistry. 91(8),5028-35.

Tsukui A., Vendramini, P.H., Garrett, R., Scholz, M.B.S., Eberlin, M.N., Bizzo, H.R., et al. 2019. Direct-infusion electrospray ionization-mass spectrometry analysis reveals atractyligenin derivatives as potential markers for green coffee postharvest discrimination. LWT. 103,205-11.

Týčová A., Ledvina, V., Klepárník, K. 2017. Recent advances in CE-MS coupling: Instrumentation, methodology, and applications. Electrophoresis. 38(1), 115-34.

Van Eeckhaut A., Lanckmans, K., Sarre, S., Smolders, I., Michotte, Y. 2009. Validation of bioanalytical LC-MS/MS assays: evaluation of matrix effects. Journal of Chromatography B. 877(23),2198-207. 
Rec. Pharm. Biomed. Sci. A: 4 (2), 24-31, 2020

van Geenen F.A.M.G., Franssen, M.C.R., Schotman, A.H.M., Zuilhof, H., Nielen, M.W.F. 2017. Ambient Characterization of Synthetic Fibers by Laser Ablation Electrospray Ionization Mass Spectrometry. Analytical Chemistry. 89(7),4031-7.
Xu R.N., Fan, L., Rieser, M.J., El-Shourbagy, T.A. 2007. Recent advances in high-throughput quantitative bioanalysis by LC-MS/MS. Journal of pharmaceutical and biomedical analysis. 44(2),34255 . 